\section{Peroral endoscopic myotomy using the posterior approach in an 11-month-old girl with achalasia, severe malnutrition, and recurrent pneumonia}

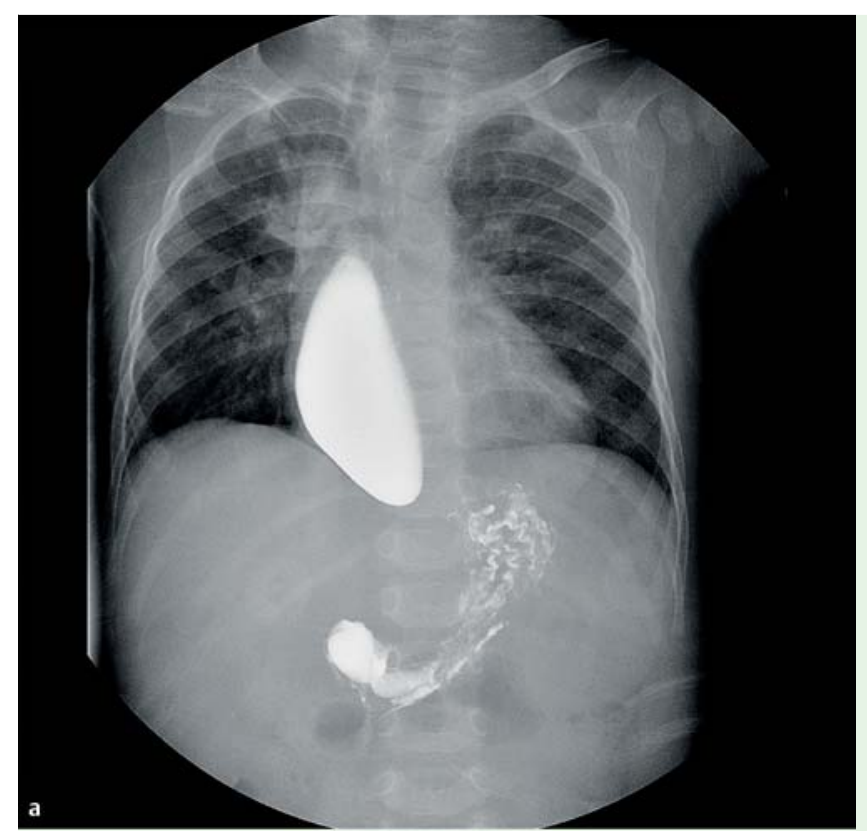

Fig. 1 Esophagography in an 11-month-old girl with achalasia, severe malnutrition, and recurrent pneumonia. a Before peroral endoscopic myotomy (POEM), showing the "bird beak" appearance. $\mathbf{b}$ At day 166 postPOEM showing smooth passage of contrast into the stomach.

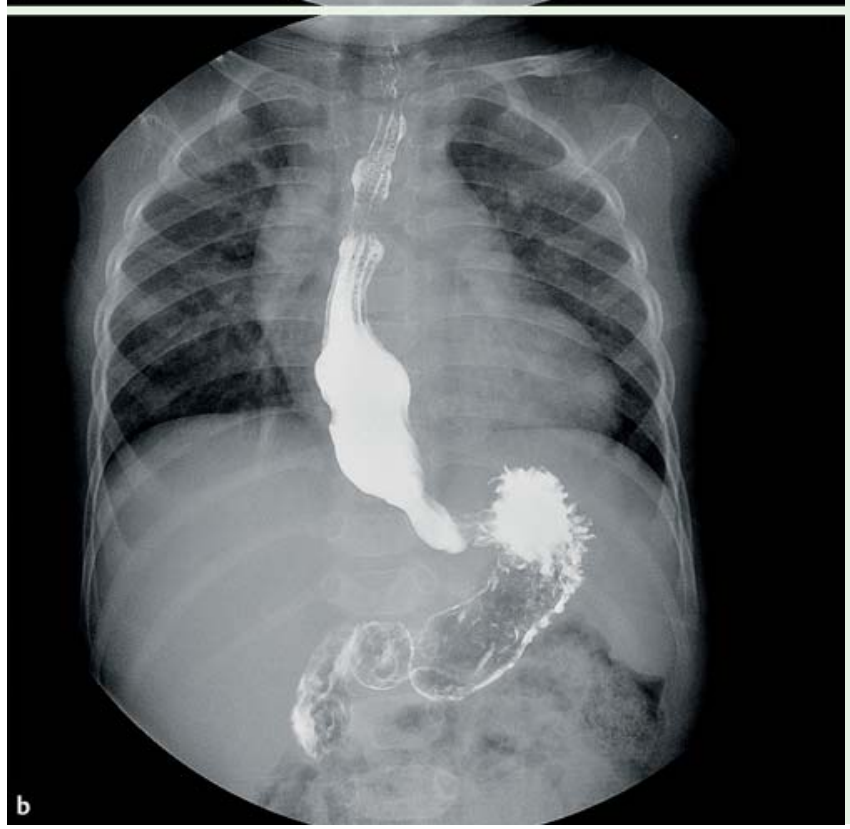

Peroral endoscopic myotomy (POEM) has become widely accepted as a minimally invasive procedure for treatment of achalasia. Here we report a case of achalasia in an 11-month-old girl that was managed by POEM.

The patient had been delivered at full term and weighed $2.95 \mathrm{~kg}$ at birth. Her growth and development were normal until she was 5 months old, when she developed nonprojectile vomiting after feeding. She began to have intermittent cough with fever 3 months later and aspiration pneumonia was suspected. Esophagography showed narrowing of the esophageal gastric junction (EGJ) ( $\bullet$ Fig.1a), that was confirmed by endoscopy. A diagnosis of achalasia was made. Manometry was not performed because of the young age of the patient. At presen- tation to us the 11-month-old patient weighed only $7.3 \mathrm{~kg}$, below the $3 \mathrm{rd}$ percentile for her age. POEM was planned. She stayed in hospital for 9 days before the procedure, undergoing nasogastric tube feeding and antibiotic treatment for pneumonia.

POEM was then performed by an experienced endoscopist (P.H.Z) ( $\bullet$ Fig. 2 , - Video 1). The standard procedure for adult patients was used, with a standard gastroscope (GIF-Q260J; Olympus, Tokyo, Japan), a hybrid knife (ERBE; Erbe Elektromedizin, Tübingen, Germany) for injection, mucosal dissection, and myotomy, hot biopsy forceps (FD-410R; Olympus) for hemostasis, and clips (HX-610-90; Olympus) for mucosal closure. An initial mucosal incision was done in the 5-6 o'clock position on the posterior esophagus about $5 \mathrm{~cm}$ above the esophagogastric junction (EGJ) (incisor-cardia distance $25 \mathrm{~cm}$ ). Myotomy was begun $4 \mathrm{~cm}$ above the EGJ and extended $1 \mathrm{~cm}$ into the cardia. The infant esophageal muscle was extremely thin and fragile. We applied a method that we call the "push and pull" technique: after an initial partial-thickness cut, the tip of the hybrid knife was inserted into the muscle-serosa plane at 56 o'clock and the full-thickness muscle bundle was lifted towards the esophageal lumen while being electrically dissected (the "push"). At the cardia, because of the limited space, muscle fibers were cut from the luminal side, pulling the scope with a "floor-sweeping" action (the "pull") [1]. The procedure was uneventful, with the exception of a small mucosal injury at the lower esophagus, which was later clipped. Abdominal bloating occurred and was relieved by needle insertion.

The patient had fever and a high white cell count for 2 days after the procedure. She was managed conservatively with meropenem, intravenous immunoglobulin, and intravenous hyperalimentation. Feeding was resumed at postoperative day 5 and the infant was discharged from hospital at postoperative day 13 .

Endoscopic follow-up at postoperative day 166 showed complete healing of the mucosa ( $\bullet$ Fig.3a), and low grade esophagitis ( Fig.3b). The endoscope could be passed without resistance. Esophagography showed a slightly dilated esophagus and smooth passage of contrast with mild gastroesophageal reflux ( $\bullet$ Fig. 1 b) The patient experienced no regurgitation or persistent vomiting following the procedure. There were 2 episodes of upper 


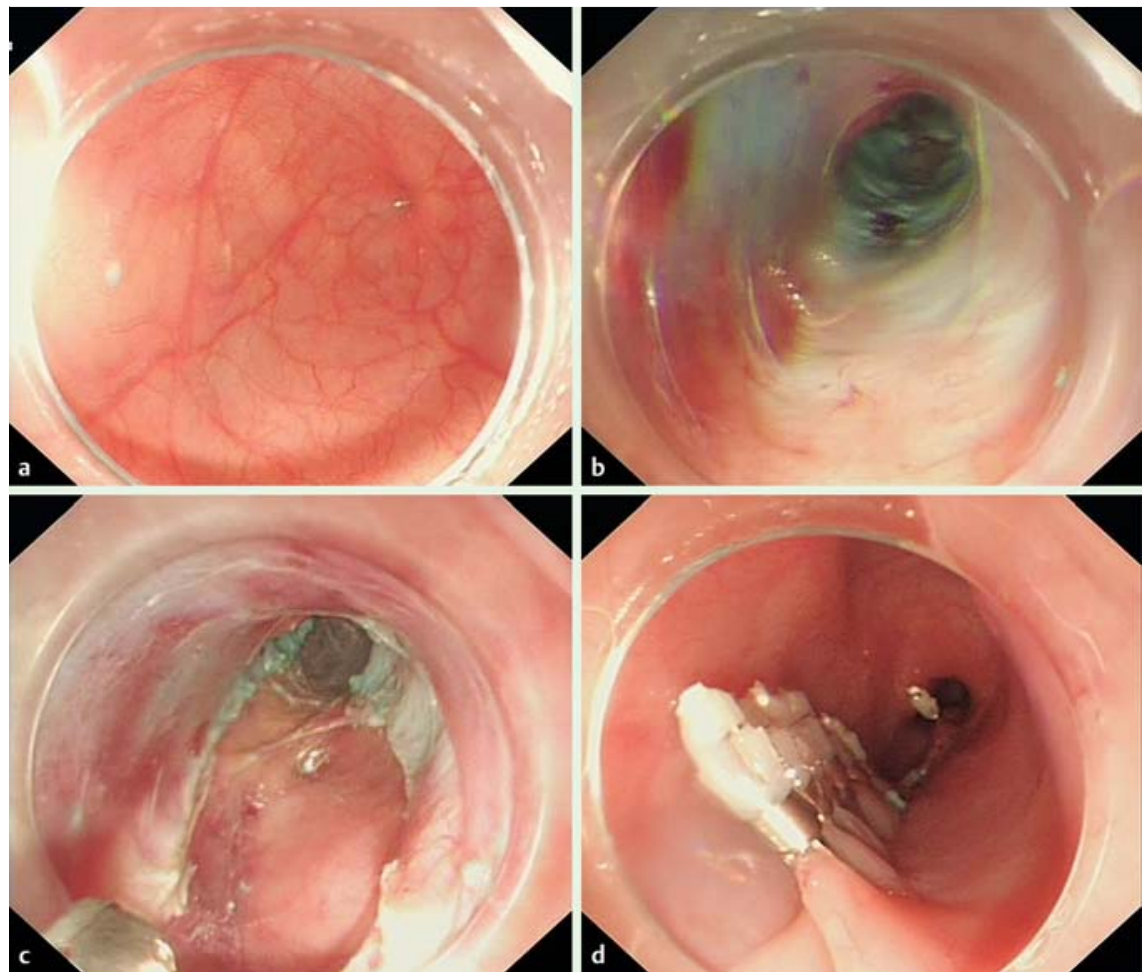

Fig. 2 Peroral endoscopic myotomy (POEM) in an 11-month-old girl with achalasia, severe malnutrition and recurrent pneumonia. a Severe narrowing of the esophagogastric junction (EGJ). b Creation of submucosal tunnel. c Submucosal full-thickness myotomy. $\mathbf{d}$ Complete closure of the mucosal flap and the mucosal injury with clips.
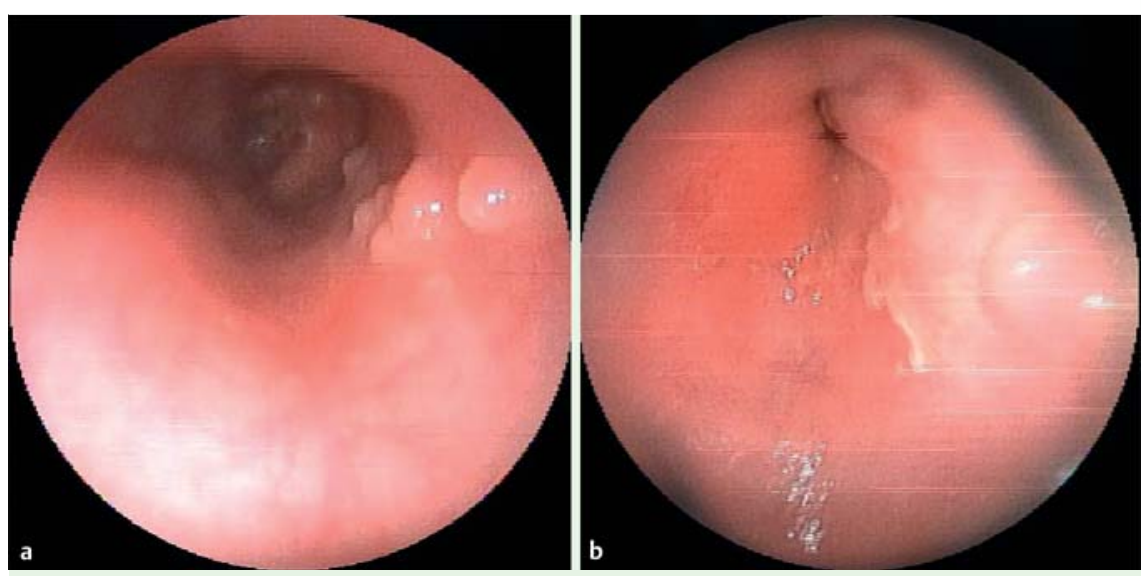

Fig. 3 Endoscopic appearance at postoperative day 166: a complete healing of mucosal flap; and b mild esophagitis.

respiratory tract infection and 2 episodes of transient diarrhea and vomiting during follow-up, all managed expectantly. No pneumonia occurred. Her weight at postoperative day 166 was $9.0 \mathrm{~kg}$.

Achalasia is rare in children, and there is no established ideal treatment [2]. Compared with adults, achalasia in young children is even more hazardous leading to recurrent aspiration and lung infection. Developmental retardation can follow. The Eckardt score [3] is not applicable in this context, and we propose the use of regurgitation, lung infection, and growth curve for evaluation of severity. POEM in small children is risky, not only because of the technical difficulty of operating on fragile tissues in a narrow space, but also because patients often present with malnutrition, immunosuppression, and chronic lung infection. Maselli et al. have previously reported successful POEM in a 3-year-old [4]. To our knowledge, however, our case is the first report of POEM in an infant. We here demonstrate that in experienced hands it is both safe and effective.

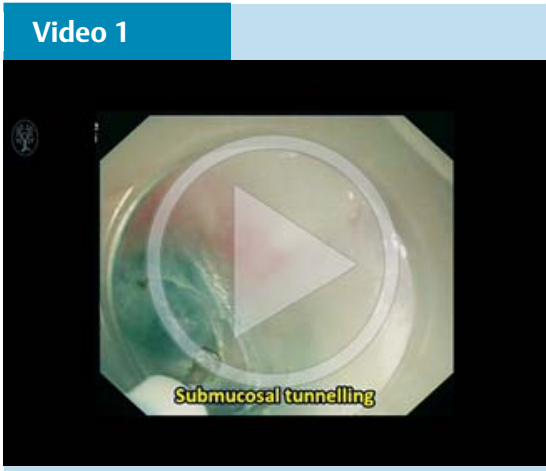

Peroral endoscopic myotomy (POEM) in an 11-month-old girl, using the "push and pull" technique.

Post-procedure reflux is a serious concern given the potential risk of long-term acid exposure in the esophagus. Close followup and optimal feeding is essential, requiring multidisciplinary consultation and good parental compliance. The present case may also indicate that the endoscopic tunneling technique is applicable in young children, and may possibly be used in diseases such as congenital hypertrophic pyloric stenosis in the future.

\section{Acknowledgments}

$\nabla$

This study was supported by grants from the Major Project of Shanghai Municipal Science and Technology Committee (13411950801), the Academic Leader Training Project of Shanghai Municipal Commission of Health and Family Planning (13B038), the National Natural Science Foundation of China (81302098, 81201902, 81470811, and 81401930), and the Natural Science Foundation of Shanghai (13ZR1452300).

Endoscopy_UCTN_Code_TTT_1AO_2AD

Competing interests: The authors declare that they have no competing interests to report with respect to this manuscript.

\section{Xiao-Cen Zhang ${ }^{1}$, Quan-Lin Li ${ }^{1}$, Ying Huang ${ }^{2}$, Shi-Jian Miao², Ping-Hong Zhou ${ }^{1}$}

1 Endoscopy Center and Endoscopy Research Institute, Zhongshan Hospital, Fudan University, Shanghai, China 2 Department of Gastroenterology, Children's Hospital, Fudan University, Shanghai, China 


\section{References}

1 Li QL, Zhou PH. Perspective on peroral endoscopic myotomy for achalasia: Zhongshan experience. Gut Liver 2015; 9: 152 - 158

2 Sharp NE, St Peter SD. Treatment of idiopathic achalasia in the pediatric population: a systematic review. Eur J Pediatr Surg 2015: Epub 2015/02/03

3 Eckardt AJ, Eckardt VF. Treatment and surveillance strategies in achalasia: an update. Nat Rev Gastroenterol Hepatol 2011; 8: $311-319$

4 Maselli R, Inoue $H$, Misawa $M$ et al. Peroral endoscopic myotomy (POEM) in a 3-yearold girl with severe growth retardation, achalasia, and Down syndrome. Endoscopy 2012; 44 (Suppl. 02): E285 -E287

\section{Bibliography}

DOI http://dx.doi.org/

10.1055/s-0034-1393156

Endoscopy 2015; 47: E480-E482

(C) Georg Thieme Verlag KG

Stuttgart · New York

ISSN 0013-726X

\section{Corresponding author}

\section{Ping-Hong Zhou, MD, PhD}

Endoscopy Center and Endoscopy Research Institute

Zhongshan Hospital, Fudan University

180 Fenglin Road

Shanghai, 200032

P. R. China.

Fax: +86-21-64038472

zhou.pinghong@zs-hospital.sh.cn 